\title{
Análisis termoenergético del sistema de generación de vapor de una central térmica de $49 \mathrm{MW}$
}

\section{(Thermo-energetic analysis of the steam generation system in a $49 \mathrm{MW}$ power plant)}

Yoalbys Retirado Mediaceja1, Héctor Luis Laurencio Alfonso², Andres A. Sánchez- Escalona, Yanán Camaraza-Medina ${ }^{4}$, Marcelo Fabián Salazar Corrales ${ }^{5}$, Marbelis Lamorú Urgelles ${ }^{6}$, Ever Góngora Leyva ${ }^{6}$

\section{Resumen}

En el trabajo se realiza un análisis termoenergético integral del sistema de generación de vapor de una termoeléctrica de 49 MW, lo cual no ha sido estudiado con rigor desde el punto de vista energético. En un algoritmo se sintetiza la metodología para el cálculo de los rendimientos térmicos brutos y exergéticos de las calderas que lo integran, aspectos que no se han interrelacionado en estudios precedentes. Los resultados evidencian un elevado grado de aprovechamiento de la energía térmica y una baja capacidad de utilización de la exergía disponible en las instalaciones, provocados por el deterioro de algunos de sus parámetros de operación y por irreversibilidades inherentes al proceso de transformación del agua en vapor. Los rendimientos, térmico y exergético del sistema, ascendieron a 90.106 y $45.491 \%$, respectivamente. El algoritmo propuesto prevé el cálculo y la comparación de los parámetros termoenergéticos reales de las calderas con los nominales y las acciones científicas, y técnico-organizativas, a desarrollar para lograr rendimientos termoexergéticos superiores. Su implementación futura tendrá en cuenta el análisis de los rendimientos térmicos netos, los indicadores económico-ambientales y la optimización energéticooperacional de las calderas.

\section{Palabras clave}

Calderas; eficiencia; térmico y exergético; algoritmo; generadores de vapor.

\begin{abstract}
In the work carries out a comprehensive thermo-energetic analysis of the steam generation system of a $49 \mathrm{MW}$ thermal power plant, which has not been rigorously studied from an energy point of view. In a algorithm synthesizes the methodology for calculating the gross thermal and exergetic performances of the boilers that comprise it, aspects that have not been interrelated in previous studies. The results show a high degree the harnessing of thermal energy and a low capacity for using the exergy available in the facilities, caused by the deterioration of some of its operationals parameters and by irreversibilities inherent in the process of transforming water into steam. The thermal and exergetic yields of the system amounted to 90.106 and $45.491 \%$, respectively. The proposed algorithm foresees the calculation and the comparison of the real thermo-energetic parameters of the boilers with the nominal ones and the scientific and technical-organizational actions to develop out to achieve superior thermo-exergetic performances. Its future implementation will take into account the analysis of the net thermal yields, the economicenvironmental indicators and the energy-operational optimization of the boilers.
\end{abstract}

\section{Keywords}

Boilers; efficiency; thermal and exergetic; algorithm; steam generators.

\footnotetext{
$1 \quad$ Universidad de Moa, Moa-Cuba (yretirado@ismm.edu.cu). https://orcid.org/0000-0002-5098-5675.

$2 \quad$ Universidad Técnica de Cotopaxi, Latacunga-Ecuador (hector.laurencio@utc.edu.ec).

3 Empresa Moa Nickel S.A.- Pedro Sotto Alba, Moa-Cuba (aescalon@moanickel.com.cu).

$4 \quad$ Universidad de Matanzas, Matanzas-Cuba (yanan.camaraza@umcc.cu).

$5 \quad$ Universidad de las Fuerzas Armadas, Latacunga-Ecuador (mfsalazar@espe.edu.ec).

$6 \quad$ Universidad de Moa, Moa-Cuba (\{mlamoru, egongora\}@ismm.edu.cu).
} 


\section{Introducción}

Los generadores de vapor son instalaciones esenciales en el funcionamiento de prácticamente todas las empresas industriales. Por su importancia se consideran el corazón de toda industria moderna (Kitto y Stultz, 2015; Rubio, 2015). Estos equipos también se conocen comúnmente como calderas de vapor y se clasifican entre los intercambiadores de calor más difundidos y utilizados, de los encontrados en la técnica moderna (Camaraza, 2017). Su estudio, desde el punto de vista de la termotransferencia, resulta interesante porque en ellos se manifiestan todos los modos de transmisión del calor (Camaraza, 2018). Además, consumen gran cantidad de combustibles y contaminan el medio ambiente. Por ello se requiere explotarlos eficientemente (Buecker, 2012).

En las grandes empresas de la producción y los servicios, así como en las centrales termoeléctricas (CTE) las calderas juegan un rol más relevante (Martha de Souza, 2012), por su gran tamaño, productividad y consumos de portadores energéticos (agua, combustible y electricidad, entre otros). En ocasiones solo se dispone de un equipo, pero en la mayoría de los casos las instalaciones de calderas están dotadas de dos o más generadores de vapor. Esta realidad, casi generalizada, exige la operación eficiente de cada equipo y del sistema de generación de vapor (SGV) en su conjunto.

Las instalaciones investigadas en el presente trabajo (cuatro calderas) constituyen puestos clave en la gestión energética de la empresa donde están enclavadas. La entidad productiva no puede prescindir de estas, debido a que constituyen el SGV de la termoeléctrica convencional de $49 \mathrm{MW}$, que se requiere imprescindiblemente para garantizar la energía calorífica y una parte de la energía eléctrica que demanda el proceso productivo principal de la fábrica.

El análisis termoenergético de las calderas es una tarea compleja y laboriosa, debido a la gran cantidad de parámetros energéticos que caracterizan el funcionamiento de estos equipos y sus agregados. Sin embargo, resulta vital para diseñar estrategias que permitan la explotación eficiente de las instalaciones. En relación con lo anterior, un elemento importante a considerar en la operación eficiente y el análisis de los rendimientos térmicos y exergéticos de estos equipos lo constituye el agua de alimentación, su pureza y calidad (Borroto y Rubio, 2010), así como la resistencia por incrustaciones (Camaraza, 2020a), que influye en la operación del domo. Además, resultan relevantes el tipo de combustible y sus propiedades, el flujo de aire de combustión y su temperatura, entre otros parámetros.

En la literatura científica se reportan importantes estudios relacionados con el análisis termoenergético de calderas. En estos se aplican los métodos, directo e indirecto, de balance térmico y la modelación matemática para el cálculo del rendimiento (Patro, 2015; Harish y Baldi, 2018; Cortés, Cornes, Alomá y González, 2019), y se implementa la simulación computacional y la optimización para la evaluación energética y la selección de calderas industriales (Flynn, 2013; Hasnain et al., 2020). Asimismo, se reportan trabajos orientados a incrementar la eficiencia energética de las instalaciones mediante: el desarrollo de métodos de diagnóstico de fallos más eficientes (Rodríguez-Ramos y Llanes-Santiago, 2014), la implementación de un sistema para el control de la calidad de los gases de combustión (Pérez, Cordovés y Terán, 2016), y la optimización aplicando un sistema de gestión de energía basado en la norma ISO 50001 (Valencia, Piero y Campos, 2019).

Relacionado con el balance exergético, Jiménez, Jarquin, Durán y García (2011) analizan una caldera de 350 MW, operando a diferentes cargas (100; 75; 50 y 25 \%). Para ello realizaron 
un modelo considerando el equipo como un volumen de control y demostraron que la generación de irreversibilidades se maximiza cuando la caldera trabaja a cargas menores (50 y $25 \%$ ). Krasniqi-Alidema, Filkoski y Krasniqi (2018) desarrollan un modelo de optimización para determinar la disipación de exergía en los componentes de una CTE de 315 MW, concluyendo que la mayor destrucción de exergía ocurre en la caldera, debido a irreversibilidades de los procesos de combustión y transferencia de calor entre los gases y la mezcla agua-vapor. A estos aspectos también se han referido otros autores (Jiménez, Madrigal, Lapido y Vidal, 2016; Velázquez, Corrales y Pérez, 2019).

Como se aprecia, existen varias publicaciones que abordan aspectos específicos relacionados con el análisis termoenergético de los generadores de vapor que operan en el sector industrial y las CTE. Sin embargo, los estudios previos consultados no establecen un algoritmo que integre las metodologías establecidas para el balance termoexergético de estas instalaciones. En general, se limitan a la exposición y aplicación de las ecuaciones propuestas para el cálculo de los rendimientos, térmico y exergético, de las calderas. En la presente investigación resulta novedoso la sistematización de los procedimientos establecidos en trabajos precedentes y su integración en un algoritmo, en el cual se establece la secuencia de pasos a seguir y los parámetros que se requieren para la evaluación térmica (por los métodos directo e indirecto), y exergética, de los generadores de vapor estudiados y otros de similares características.

Teniendo en cuenta los aspectos anteriores, se plantea como objetivo del presente trabajo realizar el análisis termoenergético del sistema de generación de vapor investigado, para las condiciones actuales de explotación. Y sus aportes más significativos se sintetizan en el algoritmo que sistematiza la metodología establecida para el balance térmico y exergético de calderas de vapor convencionales (acuotubulares y pirotubulares); la determinación de los principales parámetros termoenergéticos de las instalaciones; y las acciones científicas y técnico-organizativas que contribuyen a incrementar los rendimientos térmicos y exergéticos de las calderas.

En las secciones restantes del trabajo se exponen los métodos de balance térmico y exergético, el algoritmo mediante el cual se sistematiza la metodología establecida y los datos experimentales usados en la evaluación termoenergética de las calderas y el sistema de generación de vapor. Luego, se discuten los resultados obtenidos en los balances (térmico y exergético) realizados, se arriba a conclusiones sobre los aspectos más relevantes abordados y se expone sobre las líneas futuras de investigación.

\section{Materiales y métodos}

La evaluación termoenergética del SGV tiene en cuenta los procedimientos establecidos para balance térmico, métodos directo e indirecto, y exergético, de las calderas que lo integran (Jiménez et al., 2016; Pérez-Sánchez, Fernández y González, 2017). También considera diversos aspectos teóricos relevantes para este análisis (Annaratone, 2019). Su adecuación a las instalaciones estudiadas se expone a continuación.

\subsection{Balance térmico: métodos directo e indirecto}

El método directo consiste en medir un grupo de parámetros de operación directamente en las calderas y con ellos determinar la eficiencia térmica bruta de las instalaciones, mediante la ecuación 3 de la figura 1 (Ganapathy, 2013; Rastogi, Sharma y Gupta, 2018). 
El calor útil $\left(Q_{u u t i l}\right)$ se calcula por la ecuación 1 de la figura 1, considerando que en las calderas solo se produce vapor sobrecalentado. Para el calor disponible $\left(Q_{\mathrm{d}}\right)$, se calcula el valor calórico inferior del combustible $\left(Q_{b}\right)$, mediante la fórmula de Mendeleev (Rubio, 2015). La misma posibilita determinar la exergía química del combustible, como una función de su composición química elemental (Peduzzi, Boissonnet y Maréchal, 2016).

Por su parte, en el método indirecto se determinan las pérdidas de calor presentes en las calderas y con estas se calcula la eficiencia térmica bruta de las instalaciones, a través de las ecuaciones 4 y 5 de la figura 1 (Valles, Acosta y Pérez, 2014; Rubio, 2015).

La pérdida de calor con los gases de salida $\left(q_{2}\right)$, por incombustión química $\left(q_{3}\right)$, por incombustión mecánica $\left(q_{4}\right)$, por transferencia térmica al medio ambiente $\left(q_{5}\right)$, con los residuos extraídos del horno $\left(q_{6}\right)$ y con las extracciones o purgas $\left(q_{7}\right)$, se calculan usando las ecuaciones propuestas por Rubio (2015). En los análisis se consideran despreciables las pérdidas por incombustión mecánica y con los residuos extraídos del horno $\left(q_{4} \gg q_{6} \gg 0\right)$, por no tener significación práctica cuando se quema combustible líquido en las calderas.

Los flujos de aire y gases trasegados por el ventilador de tiro forzado (VTF) y el ventilador de tiro inducido (VTI) se calculan con las ecuaciones 8 y 9 (Pankratov, 1987).

$$
\begin{gathered}
Q_{a}=\beta_{1} \cdot B \cdot V_{a}^{o} \cdot \alpha_{h} \cdot\left(\frac{T_{a f}+273,15}{273,15}\right) \cdot \frac{1,01 \cdot 10^{5}}{h_{b}} \\
Q_{g}=\beta_{1} \cdot B \cdot\left[V_{g}^{o}+\left(\alpha_{a s}-1\right) \cdot V_{a}^{o}\right] \cdot\left(\frac{T_{g}+273,15}{273,15}\right) \cdot \frac{1,01 \cdot 10^{5}}{h_{b}}
\end{gathered}
$$

Donde: $Q_{a}$ y $Q_{g}$ - flujos de aire y de gases trasegados por el VTF y el VTI, $\mathrm{m}^{3} / \mathrm{s} ; \beta_{1}$ - coeficiente de reserva de la alimentación $\left(\beta_{1}=1.05\right.$ para $\left.D_{\text {vsc }}>5.6 \mathrm{~kg} / \mathrm{s}\right)$, adimensional; $B$ - flujo de combustible quemado, $\mathrm{kg} / \mathrm{s} ; V^{0}{ }_{a}$ - volumen de aire teórico seco, $\mathrm{m}^{3} \mathrm{~N} / \mathrm{kg} ; \alpha_{\mathrm{h}}$ y $\alpha_{\text {as }}$ - coeficientes de exceso de aire en el hogar y antes del VTF, adimensional; $T_{a f}$ - temperatura del aire frio que entra al VTF, ${ }^{0} \mathrm{C} ; \boldsymbol{h}_{\boldsymbol{b}}$ - presión barométrica del aire, $\mathrm{Pa} ; V^{0}{ }_{g}$ - volumen total teórico de gases, $\mathrm{m}^{3} \mathrm{~N}$ / $\mathrm{kg} ; T_{g}$ - temperatura de los gases antes del VTI, ${ }^{\circ} \mathrm{C}$.

\subsection{Método exergético}

La exergía es el trabajo útil máximo que puede obtenerse de un sistema en un estado y ambiente determinados (Çengel y Boles, 2014; Bluestein, 2020). En la evaluación cuantitativa del grado de perfección o de irreversibilidad de un proceso, equipo o instalación se utiliza el concepto de rendimiento exergético. Su definición general más aceptada es la representada a través de la ecuación 12 de la figura 1 (Arshad et al., 2019; Tozlu, Büyükmurat y Özahi, 2020). Asimismo, la exergía perdida puede expresarse mediante el grado de pérdida, según la ecuación 13 de la figura 1.

En las calderas estudiadas, la exergía recuperada es la del vapor sobrecalentado $\left(E x_{\text {vsc }}\right)$ y la exergía empleada es la suma de las exergías del agua de alimentación (Ex $x_{a a}$, el combustible $\left(E x_{C}\right)$, el calor de combustión ( $\left.E x_{\mathrm{ac}}\right)$ y el aire frío $\left(E x_{\mathrm{af}}\right)$, estas se calculan por las ecuaciones propuestas por Agung et al. (2017) y Javadi et al. (2019).

Los métodos de cálculo generales establecidos en la literatura para la evaluación térmica y exergética de calderas de vapor y el procedimiento específico expuesto, para las calderas investigadas, se sintetizan y sistematizan en el algoritmo de la figura 1, el cual constituye el apor- 
te fundamental del presente trabajo. El mismo prevé el cálculo de los parámetros termoenergéticos fundamentales de las instalaciones y las acciones a implementar para lograr rendimientos térmicos y exergéticos superiores.

Figura 1. Algoritmo para la sistematización de la metodología del balance termoexergético

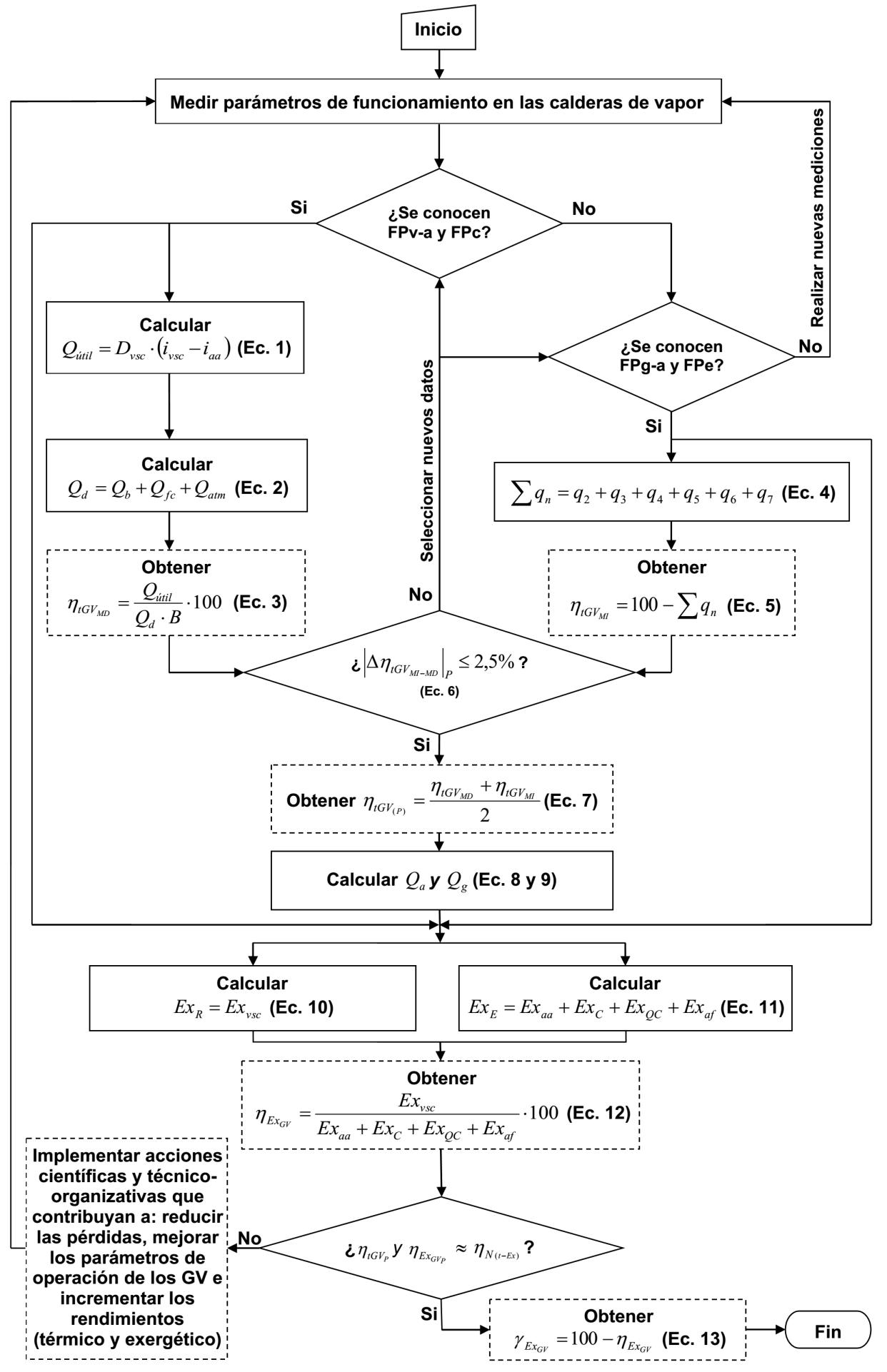


Leyenda para la figura 1: FPV-a (Flujos y propiedades termodinámicas del vapor sobrecalentado, el vapor de atomización y el agua de alimentación); FPc (Flujo y propiedades del combustible, incluye la composición química); FPg-a (Flujos, composición química y propiedades de los gases y el aire); FPe (Flujo y propiedades termodinámicas de las extracciones o purgas) y $\eta_{\mathrm{N}}$ (t:-Ex) (Rendimiento térmico o exergético establecido para las condiciones nominales de operación).

Parámetros de las ecuaciones de la figura 1: $Q_{u \text { util }}$ - calor útil, $\mathrm{kJ} / \mathrm{h} ; \mathrm{D}_{\mathrm{vsc}}$ - flujo de vapor sobrecalentado, $\mathrm{kg} / \mathrm{h} ; i_{\mathrm{vsc}}$ - entalpía del vapor sobrecalentado, $\mathrm{kJ} / \mathrm{kg}_{;} i_{\text {aа }}$ - entalpía del agua de alimentación, $\mathrm{kJ} / \mathrm{kg} ; Q_{\mathrm{d}}$ - calor disponible, $\mathrm{kJ} / \mathrm{kg} ; Q_{\mathrm{b}}$ - poder calórico inferior del combustible, $\mathrm{kJ} /$ $\mathrm{kg} ; \mathrm{Q}_{\mathrm{fc}}$ - calor físico del combustible, $\mathrm{kJ} / \mathrm{kg} ; \mathrm{Q}_{\mathrm{atm}}$ - calor que aporta el vapor de atomización, $\mathrm{kJ} /$ $\mathrm{kg} ; \eta_{t G V_{M D}}$ - rendimiento térmico bruto de la caldera (obtenido por el método directo), \%; B consumo de combustible (se mide); $\mathrm{kg} / \mathrm{h} ;$; $q_{\mathrm{n}}$ - sumatoria de las pérdidas de calor, \%; $\eta_{t G V m I}$ - rendimiento térmico bruto de la caldera (obtenido por el método indirecto), \%; $\left|\eta_{t G V M D-M I}\right|$ valor absoluto de la diferencia entre los valores de rendimiento térmico bruto obtenidos por el los métodos directo e indirecto, \%. $\eta_{t G V_{(P)}}$-rendimiento térmico bruto promedio, \%; $E x_{R} y E x_{E}$ - exergías recuperada y empleada, $\mathrm{J} / \mathrm{s} ; \eta_{E x G V}$ - rendimiento exergético del generador de vapor, $\% ; E x_{v s c}, E x_{a a}, E x_{C}, E x_{Q C}, E x_{a f}$ - exergías del vapor sobrecalentado, el agua de alimentación, el combustible, el calor de combustión y el aire frío, $\mathrm{kJ} / \mathrm{s}$; ExGV - grado de pérdida de exergía en la caldera, \%.

\subsection{Características de la CTE y sus áreas principales}

La CTE es la encargada de producir y distribuir los portadores energéticos necesarios para el proceso tecnológico. Tiene como equipos fundamentales siete calderas, dos de $150 \mathrm{t} / \mathrm{h}$ y cinco de $75 \mathrm{t} / \mathrm{h}$, todas están diseñadas para producir vapor sobrecalentado a 39 atm y $440{ }^{\circ} \mathrm{C}$. Además, consta con tres turbinas de vapor, dos de $12 \mathrm{MW}$ y una $25 \mathrm{MW}$ (49 MW total). En la actualidad la CTE se opera con un generador de vapor de 150 t/h (GV-6) y tres de 75 t/h (GV-2; G-4 y GV-5). Además, se emplean dos turbinas, una de $12 \mathrm{MW}$ y la de $25 \mathrm{MW}$. Los restantes equipos no utilizados (tres calderas y una turbina) se tienen de reserva para garantizar la permanente disponibilidad de las plantas de generación térmica y eléctrica. Las áreas fundamentales que integran la planta termoenergética son: base de petróleo, tratamiento químico de agua, calderas, turbinas y torres de enfriamiento. Las áreas de mayor influencia en el proceso de producción de vapor se describen a continuación; las restantes se detallan en la bibliografía consultada (Labañino, 2015).

\subsection{1. Área de tratamiento químico del agua}

Está formada por tres instalaciones tecnológicas: suavizamiento, desmineralización y neutralización. El agua inicial es potable y proviene de la planta potabilizadora de la comunidad, la cual llega por dos tuberías de diámetro de $300 \mathrm{~mm}$. Se somete a un proceso químico en la referida planta para eliminar las impurezas en suspensión y coloides, evitando la contaminación de las resinas en la planta, la formación de incrustaciones y las causas que atentan contra la calidad del vapor producido en la CTE. A las calderas se debe alimentar agua con las siguientes especificaciones de calidad: temperatura $\left(145^{\circ} \mathrm{C}\right)$ contenido de $\mathrm{SiO}_{2}(0.02 \mathrm{mg} / \mathrm{l})$, dureza $(5 \mu \mathrm{mol} / \mathrm{l}$ máx.), pH (9.0), conductividad eléctrica específica (0.20 $\mu \mathrm{S} / \mathrm{cm}$ máx.), contenido de $0_{2}(0.02$ mg/l máx.), oxidabilidad como $\mathrm{KMnO}_{4}(3.0 \mathrm{mg} / \mathrm{l})$ y contenido del aceite $(0.5 \mathrm{mg} / \mathrm{l})$. A la fecha, estos estándares de calidad se garantizan con estabilidad porque de lo contrario no será posible emplearla para el enfriamiento de vapor en el atemperador de las calderas. 


\subsection{2. ÁREA DE CALDERAS DE VAPOR}

Debe garantizar el flujo de vapor estable al proceso productivo, con los parámetros adecuados de presión y temperatura, así como el flujo de gases producto de la combustión para la carbonatación en la planta de recuperación de amoniaco de la empresa. Las características técnicas de las calderas que se operan en la CTE y del combustible que se debe utilizar se exponen a continuación.

\begin{tabular}{|c|c|c|}
\hline \multicolumn{3}{|c|}{ Características técnicas de las calderas } \\
\hline País y año de fabricación & República Eslovaca (2007) & *URSS (1986) \\
\hline \multirow{2}{*}{ Cantidad instalada (y en operación) } & $2(1)$ & $5(3)$ \\
\hline & \multicolumn{2}{|c|}{ Parámetros nominales de diseño } \\
\hline Producción de vapor & $150 \mathrm{t} / \mathrm{h}$ & $75 \mathrm{t} / \mathrm{h}$ \\
\hline Presión del vapor sobrecalentado & 39 atm & 39 atm \\
\hline Temperatura del vapor sobrecalentado & $440^{\circ} \mathrm{C} \pm 8^{\circ} \mathrm{C}$ & $440^{\circ} \mathrm{C} \pm 8^{\circ} \mathrm{C}$ \\
\hline Temperatura de agua de alimentación & $145^{\circ} \mathrm{C}$ & $145^{\circ} \mathrm{C}$ \\
\hline \multirow[t]{2}{*}{ Eficiencia térmica bruta } & $92-93 \%$ & $90-91 \%$ \\
\hline & \multicolumn{2}{|c|}{ *Unión de Repúblicas Socialistas Soviéticas } \\
\hline
\end{tabular}

Las calderas deben operar con el combustible petróleo crudo cubano 1400, con las siguientes especificaciones técnica de calidad: viscosidad a $50{ }^{\circ} \mathrm{C}$ (859-1484 cSt), viscosidad a $80{ }^{\circ} \mathrm{C}$ (133213 cSt), peso específico a $15.5^{\circ} \mathrm{C}\left(0,9881-0.9994 \mathrm{~kg} / \mathrm{dm}^{3}\right)$, punto de inflamación en el recipiente abierto $\left(40{ }^{\circ} \mathrm{C}\right)$, carbón conradson (10.55-13.34 \%), ceniza (0.044-0.076\%), agua (1.6-2.0\%), azufre (6.33-8.0\%), vanadio (64-86 ppm), níquel (30-48 ppm), sales (310-462 ppm), asfaltenos (17.3$19.75 \%)$, hidrógeno (9.64-10.27\%), carbono (79.70- 80.95\%) y potencia calorífica inferior (9200$9208 \mathrm{kcal} / \mathrm{kg}$ ). Además, la temperatura de bombeo tiene que estar en el rango de 50 a $75^{\circ} \mathrm{C}$.

\subsection{3. ÁrEA de tURBINAS DE VAPOR}

Está equipada con tres turbinas a condensación con extracciones reguladas para el proceso (a 10.5 y 2.0 bar), así como una extracción no regulada para el calentamiento del agua de alimentación a las calderas. Sus características se exponen a continuación.

\begin{tabular}{|c|c|c|}
\hline \multicolumn{3}{|l|}{ Características técnicas } \\
\hline País y año de fabricación & República Eslovaca (2015) & *URSS (1986) \\
\hline Cantidad instalada (y en operación) & $1(1)$ & $2(1)$ \\
\hline & \multicolumn{2}{|c|}{ Parámetros nominales de diseño } \\
\hline Identificación & Skoda KP-25-3.5 & PR-12/12-3.5/1/0.2 \\
\hline Potencia & $25 \mathrm{MW}$ & $12 \mathrm{MW}$ \\
\hline Consumo máximo de vapor & $188 \mathrm{t} / \mathrm{h}$ & $110 \mathrm{t} / \mathrm{h}$ \\
\hline Presión del vapor & 35 atm & 35 atm \\
\hline Temperatura del vapor & $435^{\circ} \mathrm{C}$ & $435^{\circ} \mathrm{C}$ \\
\hline Número de revoluciones & 3600 rpm & $3600 \mathrm{rpm}$ \\
\hline
\end{tabular}

\subsubsection{Principales problemas existentes en la CTE}

A partir del diagnóstico energético realizado en la planta termoeléctrica se corroboró que en la misma existen los siguientes problemas no resueltos: 
- Trabajo por tiempo prolongado de las calderas en condiciones de explotación que difieren significativamente de las de diseño: operación a cargas parciales pequeñas que fluctúan constantemente; reducción de la calidad del vapor producido, respecto a la nominal, insuficiente calentamiento del agua suministrada al domo de las calderas; cambio de combustible sin realizar las modificaciones necesarias a los quemadores e insuficiente mantenimiento, entre otros.

- Frecuentes salidas de servicio debido a: sub y sobre nivel de agua en el domo, insuficiente temperatura del petróleo alimentado a las calderas, alta presión en el horno, ponches en los economizadores y fallos en equipos auxiliares.

- Irreversibilidades típicas de los procesos de combustión, transferencia de calor y de transformación del agua en vapor, debido a la notable diferencia de temperatura que existe entre la sustancia de trabajo y los gases de la combustión, la cual se ha acentuado por deficiencias en la operación de los economizadores.

- No implementación de acciones científicas y técnico-organizativas en la planta térmica, que contribuyan a reducir las pérdidas de calor y elevar los rendimientos térmicos y exergéticos de las instalaciones objeto de estudio.

\subsection{Parámetros de funcionamiento de las calderas}

Debido al régimen de producción continuo de las instalaciones estudiadas se aplicó un experimento pasivo. Este consiste en la observación y registro de las variables de entrada y salida del proceso en el régimen normal de trabajo del objeto investigado, sin la manipulación de las variables por parte del investigador. En su lugar, el control se ejerce sobre los niveles de los parámetros estudiados (Edmonds y Kennedy, 2017).

Durante seis semanas se tomaron 24 mediciones diarias (cada una hora) de los parámetros de operación de las calderas estudiadas en la CTE $(2 ; 4 ; 5$ y 6$)$ y se determinó el valor promedio por semana, el cual corresponde a cada una de las variantes analizadas ( $\mathrm{V}-1, \ldots, \mathrm{V}-6)$. Por razón de espacio solo se exponen los datos seleccionados de la caldera 5, ver Tablas 1 y 2. Se escoge esta instalación por tener el mejor desempeño exergético.

Tabla 1. Principales parámetros de funcionamiento medidos en las calderas seleccionadas ${ }^{1}$

\begin{tabular}{|c|c|c|c|c|c|c|c|}
\hline \multirow{2}{*}{$\begin{array}{c}\text { Parámetros } \\
\text { de la Caldera } 5\end{array}$} & \multicolumn{6}{|c|}{ Variantes consideradas (valores promedios) } & \multirow{2}{*}{ Unidades } \\
\hline & V-1 & V-2 & V-3 & V-4 & V-5 & V-6 & \\
\hline$D_{v s c}$ & 46.0 & 41.1 & 38.7 & 39.7 & 43.3 & 40.0 & $\mathrm{t} / \mathrm{h}$ \\
\hline$P_{\text {vsc }}$ & 3.80 & 3.72 & 3.68 & 3.65 & 3.65 & 3.66 & $\mathrm{MPa}$ \\
\hline$P_{D}$ & 3.90 & 3.90 & 3.99 & 3.87 & 4.00 & 3.89 & $\mathrm{MPa}$ \\
\hline $\mathrm{T}_{\mathrm{vsc}}$ & 431.4 & 312.3 & 428.8 & 440.0 & 440.0 & 437.2 & ${ }^{\circ} \mathrm{C}$ \\
\hline$t_{a a}$ & 138.7 & 142.4 & 142.7 & 131.5 & 143.0 & 142.3 & ${ }^{\circ} \mathrm{C}$ \\
\hline$T_{g s c}$ & 176.6 & 180.3 & 181.9 & 211.0 & 179.3 & 198.5 & ${ }^{\circ} \mathrm{C}$ \\
\hline$t_{c}$ & 134.6 & 136.2 & 139.6 & 138.3 & 142.0 & 142.0 & ${ }^{\circ} \mathrm{C}$ \\
\hline B & 3.6 & 3.2 & 3.0 & 3.2 & 3.4 & 3.2 & $\mathrm{t} / \mathrm{h}$ \\
\hline
\end{tabular}


Instrumentos utilizados para la medición de los parámetros de operación seleccionados (están conectados y controlados mediante el sistema SCADA):

- Flujo de vapor y agua $\left(D_{\text {vsc }}\right.$ y $D_{\text {a }}$ ): Transmisor diferencial de presión (0-100 kPa), salida 4-20 mA. Modelo EJA110AEHS5A-64NC Tag. 6LBA01F1 (Rango 0-180 t/h)

- Presión del vapor y el domo $\left(P_{\text {vsc }}\right.$ y $\left.P_{\mathrm{D}}\right)$ : Transmisor de presión relativa, salida 4-20 mA. Modelo EJA430A-EBS5A64NC. Tag-6LBA01P1 (Rango 0-6 MPa).

- Temperatura del vapor y el agua $\left(T_{\text {vsc }}\right.$ y $t_{\text {aa }}$ ): Convertidor térmico con termopar $1 \times$ NiCr-Ni "K" EL= 2400 mm, D = $6 \mathrm{~mm}$, transmisor programable, salida 4-20 mA. Modelo dTANS T01, número 956550/999-888-888 (Rango $\left.0-600^{\circ} \mathrm{C}\right)$.

- Temperatura de los gases y el aire $\left(T_{\text {gsc }} y T_{\text {af }}=30-33^{\circ} \mathrm{C}\right)$ : Convertidor térmico PT100 simple en barra, $\mathrm{D}=15 \mathrm{~mm}$, salida 4-20 mA. Modelo dTANS T01 (Rango 0-300 ${ }^{\circ} \mathrm{C}$ ).

- Flujo de combustible (B): Transmisor de peso flujómetro. Alimentación 230 V AC, salida 4-20 mA. Tag 6HJF10F1 (Rango 0-15000 kg/h).

1. Leyenda: $P_{\mathrm{vsc}}$ y $T_{\mathrm{vsc}}$ (Presión y temperatura del vapor sobrecalentado); $P_{\mathrm{D}}$ (Presión en el domo); $t_{\mathrm{ad}} T_{\mathrm{gsc}}$ y $t_{\mathrm{c}}$ (Temperatura del agua de alimentación, de los gases y del combustible).

La composición química del petróleo, en masa principal, se obtuvo en el laboratorio especializado de la empresa. Los análisis químicos se desarrollaron por métodos ASTM (American Society for Testing and Materials) estandarizados, ver tabla 2. Las calderas quemaron el mismo tipo de combustible (petróleo crudo cubano, de alto contenido de azufre), debido a que el tanque de almacenamiento y la tubería de suministro son comunes para todas. Además, se midió la composición química de los gases producto de la combustión, para ello se emplearon los instrumentos que se exponen en la siguiente tabla.

Tabla 2. Valores promedios de la composición química del combustible y de los gases

\begin{tabular}{|c|c|c|c|c|c|c|c|c|c|}
\hline \multirow{2}{*}{$\begin{array}{l}\text { Variantes } \\
\text { analizadas }\end{array}$} & \multicolumn{6}{|c|}{ Combustible, petróleo crudo (\%) } & \multicolumn{3}{|c|}{ Gases de la combustión (\%) } \\
\hline & $C^{t}$ & $\mathrm{H}^{\mathrm{t}}$ & $\mathrm{O}^{\mathrm{t}}$ & $S^{t}$ & $\mathbf{N}^{t}$ & $\mathbf{W}^{\mathrm{t}}$ & $\mathrm{CO}_{2}$ & $\mathrm{O}_{2}$ & $\mathrm{CO}$ \\
\hline V-1 & 84.10 & 7.73 & 0.6 & 6.52 & 0.2 & 0.8 & 15.9 & 2.8 & 0.17 \\
\hline V-2 & 83.91 & 7.71 & 0.6 & 6.48 & 0.2 & 0.8 & 15.1 & 2.6 & 0.17 \\
\hline V-3 & 83.51 & 7.69 & 0.7 & 6.47 & 0.2 & 0.8 & 15.8 & 2.9 & 0.19 \\
\hline$V-4$ & 83.67 & 7.68 & 0.6 & 6.51 & 0.2 & 0.8 & 15.7 & 2.4 & 0.14 \\
\hline V-5 & 83.95 & 7.59 & 0.7 & 6.49 & 0.2 & 0.8 & 15.1 & 2.5 & 0.11 \\
\hline V-6 & 83.66 & 7.61 & 0.6 & 6.43 & 0.2 & 0.8 & 15.4 & 2.6 & 0.16 \\
\hline \multicolumn{10}{|c|}{$\begin{array}{l}\text { Métodos de ensayo aplicados para el análisis del combustible (parámetro determinado): } \\
\left.\text { NC ASTM D-95 (Agua); ASTM D-129 (Azufre) y ASTM D-4698 ( } Q_{b}=9200-9300 \mathrm{kcal} / \mathrm{kg}\right) \text {. }\end{array}$} \\
\hline \multicolumn{10}{|c|}{$\begin{array}{l}\text { Instrumentos utilizados para la medición de la composición química de los gases: } \\
\text { Analizadores de gas continuos ULTRAMAT } 23 \text { (se usan dos y se toma el valor promedio), alimentación } 230 \text { V CA, } \\
\text { salida 4-20 mA. Modelo 7MB2335-2CP10-3AA1-Z-A31 (Rangos: CO, 0-250 mg/m³ } \mathrm{NO}^{3}, 0-250 \mathrm{mg} / \mathrm{m}^{3} ; \mathrm{SO}_{2^{\prime}} \text { 0-400 } \\
\mathrm{mg} / \mathrm{m}^{3} ; \mathrm{O}_{2^{\prime}} \text { 0-25\%; } \mathrm{CO}_{2^{\prime}} \text { 0-50\%. }\end{array}$} \\
\hline
\end{tabular}

En el balance termoexergético de las calderas se usó el software Mathcad15 y la aplicación informática "SteamTable" para calcular las entalpías y entropías del agua y el vapor. Las entalpías de los gases de la combustión se obtuvieron según Pankratov, 1987. 


\section{Resultados y discusión}

Los valores de los parámetros fundamentales de las calderas y el SGV, obtenidos en la evaluación termoexergética, se exponen de forma resumida en la tabla 3 y la figura 2. Los rendimientos térmicos (89.717; 90.979 y 90.602\%) y exergéticos $(45.885 ; 45.926$ y $46.076 \%)$ obtenidos para las calderas 2; 4 y 5 son semejantes, la oscilación fue de 1.262 y $0.586 \%$. Estos, analizados integralmente, permiten corroborar que los generadores de vapor tienen adecuados rendimientos térmicos, a pesar de tener 34 años de explotación y en la actualidad trabajar solo en determinadas épocas del año. Su puesta en marcha depende de las exigencias del proceso productivo de la empresa y de la no disponibilidad del GV-7, el cual tienen características técnicas similares al GV-6.

En el caso de la caldera 6, los resultados evidencian un deterioro más pronunciado de sus parámetros de funcionamiento. La producción de vapor, su presión y temperatura fueron inferiores en $37.1 \mathrm{t} / \mathrm{h} ; 0.386 \mathrm{MPa}$ y $0.483^{\circ} \mathrm{C}$, respecto a los nominales (150 t/h; $3.92 \mathrm{MPa}$ y 440 $\left.{ }^{\circ} \mathrm{C}\right)$. Lo anterior, unido a la influencia de otras variables del proceso, limita la cantidad y calidad del vapor entregado a las turbinas e influye en los rendimientos, térmico y exergético, de la CTE y la instalación productora de vapor, los cuales en esta última ascendieron a 89.125 y $45.099 \%$. Ambos son inferiores (en 2.809 y $2.531 \%$ ) a los calculados para esta caldera (91.934 y 47.630 $\%)$, en condiciones muy similares a las de diseño (149.8 t/h; $3.93 \mathrm{MPa}$ y $440.3^{\circ} \mathrm{C}$ ). En estos resultados incidió el tiempo de servicio de la caldera, puestas en marcha desde el año 2007.

Los rendimientos exergéticos de las calderas son bajos, oscilan entre el 45.099 y el $46.076 \%$. No obstante, se corresponden con los resultados esperados para estos tipos de generadores de vapor cuando se explotan a cargas parciales, sin calentamiento del aire de combustión y se obtiene vapor con una calidad (presión y temperatura) inferior a la nominal (Labañino, 2015). El valor de la exergía del vapor, que corresponde a la exergía recuperada $\left(E X_{R}\right)$ en las instalaciones, se contrajo desde los valores nominales de $24.87 \mathrm{MJ} / \mathrm{s}$ (para las calderas $2 ; 4$ y 5 ) y 49.75 MJ/s (para el GV-6) hasta los referenciados en la Tabla 3.

Tabla 3. Rendimiento térmico, exergético y grado de pérdida de exergía.

\begin{tabular}{|c|c|c|c|c|c|}
\hline Parámetros & GV-2 & GV-4 & GV-5 & GV-6 & Unidades \\
\hline$\left(\eta_{\mathrm{tGVMD}}+\eta_{\mathrm{tGVM}}\right) / 2$ & 89.717 & 90.979 & 90.602 & 89.125 & $\%$ \\
\hline $\mathrm{Ex}_{\mathrm{vsc}} \approx \mathrm{Ex}_{\mathrm{R}}$ & 17.55 & 16.95 & 13.62 & 37.04 & $\mathrm{MJ} / \mathrm{s}$ \\
\hline $\mathrm{Ex}_{9}$ & 7.925 & 5.778 & 4.385 & 23.218 & $\mathrm{MJ} / \mathrm{s}$ \\
\hline$E x_{g} / E X_{E}$ & 20.541 & 15.641 & 14.834 & 28.269 & $\%$ \\
\hline$E X_{E}$ & 38.58 & 36.94 & 29.56 & 82.13 & $\mathrm{MJ} / \mathrm{s}$ \\
\hline$\eta_{E \times(G V)}$ & 45.490 & 45.885 & 46.076 & 45.099 & $\%$ \\
\hline$\gamma_{E x(G)}$ & 54.510 & 54.115 & 53.924 & 54.901 & $\%$ \\
\hline
\end{tabular}

Como se aprecia en la figura 2, el combustible y el aire frío no aportan grandes cantidades de exergía a las calderas, sus valores promedios para el SGV ascienden a 418 y $763 \mathrm{~kJ} / \mathrm{s}$. Sin embargo, es importante considerarlos en la determinación de la exergía empleada porque eleva la precisión en el cálculo exergético y el rigor en el análisis. 
En la pérdida de exergía impactan significativamente los gases, debido a sus flujos y temperaturas a la salida de los generadores de vapor. Su exergía en las calderas 2; 4; 5 y 6 representan el 20.541; 15.641; 14.834 y 28.269 \% de la exergía empleada promedio (de las seis variantes calculadas) en cada una de las instalaciones. Y en SGV constituye el 22.064 y $40.478 \%$ de las exergías empleada y perdida, respectivamente. Las extracciones o purgas realizadas en el domo no tienen gran influencia en el parámetro considerado; su valor de exergía para el sistema apenas alcanza los $113.8 \mathrm{~kJ} / \mathrm{s}$, ver figura 2.

Al analizar de manera conjunta los resultados obtenidos, se infiere que las calderas 4 y 5 son las más eficientes desde el punto de vista térmico y exergético, por ese orden, mientras que el GV-6, a pesar de ser más moderno, tiene rendimientos térmicos y exergéticos más bajos. Esto puede estar asociado al cambio de combustible respecto al previsto en su diseño (sin la debida modificación de los quemadores) y a los problemas que ha presentado en la cámara de combustión y el horno (suciedad en las superficies de transferencia de calor, corrosión y deterioro de los tubos de agua en los economizadores, entre otros), que han limitado el aprovechamiento del calor en el hogar y otras zonas de intercambio térmico, provocando que la temperatura de los gases a la salida sea 102.63 y $115.63^{\circ} \mathrm{C}$ más alta que los valores preestablecidos (188 y 175 $\left.{ }^{\circ} \mathrm{C}\right)$ para las cargas nominal $(150 \mathrm{t} / \mathrm{h})$ y parcial de $120 \mathrm{t} / \mathrm{h}\left(0.8 \cdot \mathrm{D}_{\mathrm{N}}\right)$. Para la carga real actual de la caldera $\left(112,9 \mathrm{t} / \mathrm{h}\right.$, el $\left.0.75 \cdot D_{\mathrm{N}}\right)$ la temperatura de los gases debería ser inferior a $175^{\circ} \mathrm{C}$ y superior a $162{ }^{\circ} \mathrm{C}$ (este último es el valor límite para $0.5 \cdot D_{N}$ ) y su valor promedio fue de $290.63^{\circ} \mathrm{C}$.

En general, en el SGV existe un alto grado de aprovechamiento de la energía térmica disponible. Los rendimientos térmicos brutos obtenidos por los métodos directo e indirecto alcanzan el 91.047 y $89.164 \%$, respectivamente. Y su valor promedio fue de $90.106 \%$. Sin embargo, su rendimiento exergético fue $45.491 \%$, considerado bajo para estas calderas.

En las instalaciones y en el SGV existen reservas energéticas que no han sido suficientemente aprovechadas, relacionadas con el trabajo de las máquinas térmicas en condiciones nominales o a cargas parciales superiores a las actuales; y con las pérdidas que se producen con los gases que salen por la chimenea $\left(q_{2}\right)$ y por transferencia de calor al medio ambiente $\left(q_{5}\right)$. Estas pérdidas fueron las que más afectaron el desempeño energético de los equipos. También incidió negativamente el insuficiente calentamiento que recibió el agua de alimentación en los economizadores; $3.837^{\circ} \mathrm{C}$ menos que el valor de diseño $\left(145^{\circ} \mathrm{C}\right)$ y la contracción que experimentó el calor útil, respecto a los valores que debe tener en las calderas $2 ; 4$ y $5\left(2.024 \cdot 10^{11} \mathrm{~J} / \mathrm{h}\right)$ y en el GV-6 (4.048·10 $\left.{ }^{11} \mathrm{~J} / \mathrm{h}\right)$, cuando estas operan con los parámetros nominales (75 o $150 \mathrm{t} / \mathrm{h}$; $440{ }^{\circ} \mathrm{C}$ y $\left.3.92 \mathrm{MPa}\right)$.

El resumen de los resultados obtenidos para los parámetros termoenergéticos fundamentales del SGV se muestra en la figura 2. Este constituye la segunda contribución de la presente investigación. 
Figura 2. Diagrama de flujo termoenergético para el sistema de generación de vapor

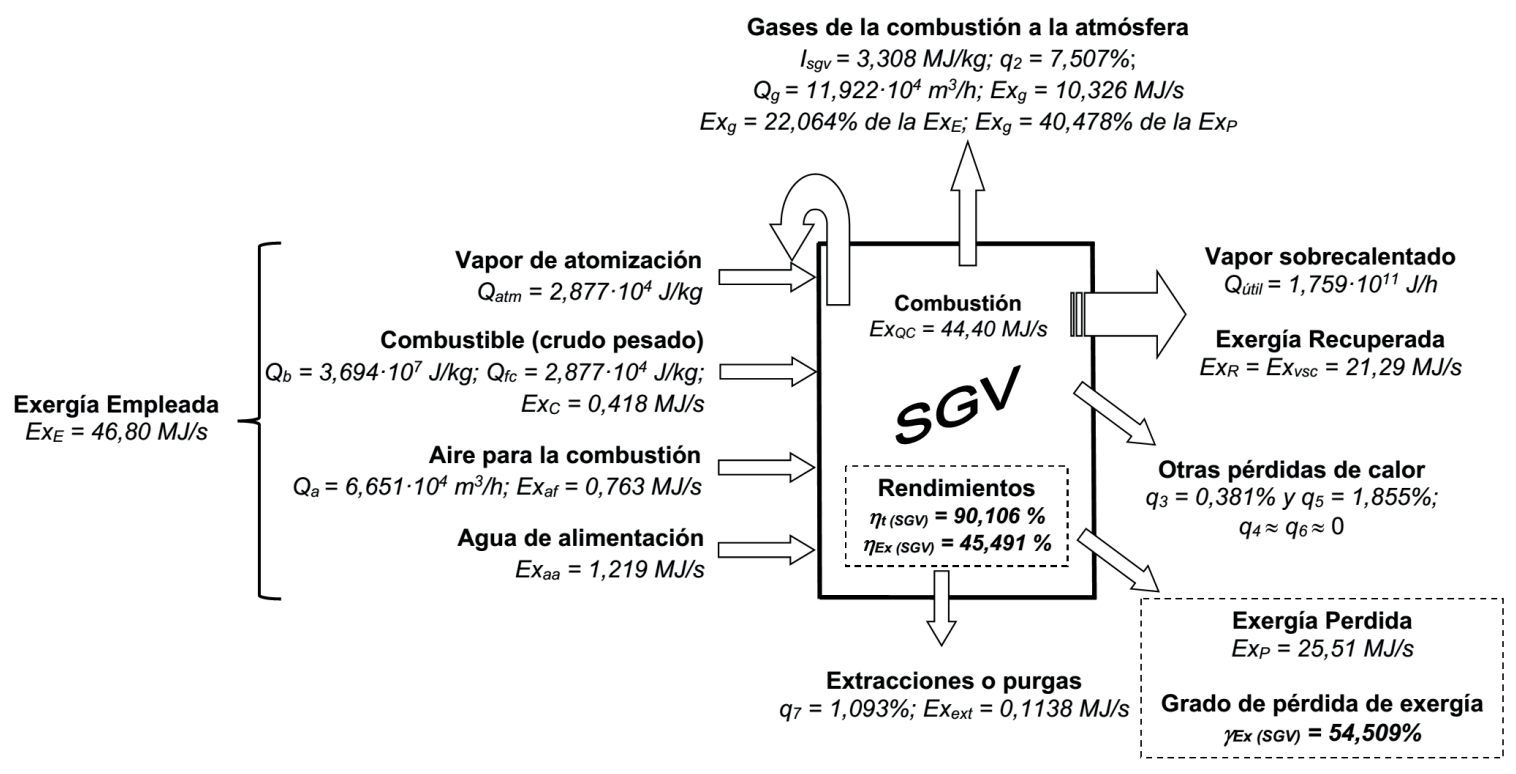

\subsection{Acciones para aumentar las eficiencias térmicas y exergéticas de las calderas}

Para mitigar y/o eliminar los problemas existentes en la CTE, perfeccionar la explotación de los equipos, aumentar la seguridad de su trabajo y optimizar su desempeño energético, se recomienda implementar integralmente las acciones siguientes:

- Diagnosticar a las calderas con rigor científico, desde el punto de vista térmico, exergético y de mantenimiento, lo cual dotará a los decisores de la empresa de la información necesaria y precisa sobre el estado de los principales parámetros de funcionamiento de las instalaciones. Y posibilitará la planificación y desarrollo de un sistema de mantenimiento que eleve su disponibilidad y fiabilidad.

- Optimizar el sistema de gestión de la energía en los generadores de vapor mediante la aplicación de la norma ISO 50001 (2018), lo cual permitirá monitorear y evaluar el proceso de producción de vapor y sus oportunidades de mejora, con referencia a sus características claves y las políticas energéticas de la empresa y el país.

- Explotar las calderas a su productividad nominal (siempre que sea posible), para mitigar y/o eliminar las ineficiencias asociadas a la subutilización de su capacidad, reducir la probabilidad de ocurrencia de roturas de carácter grave y no comprometer excesivamente la seguridad durante su operación.

- Precalentar el aire y suministrar las cantidades necesarias, para optimizar la combustión e incrementar la exergía disponible. Esta acción, el ámbito de los combustibles fósiles, requiere de un riguroso análisis de la transferencia de calor en presencia de reacciones químicas para mejorar la eficiencia de la combustión en hornos y calderas (Centeno-González et al., 2017, Camaraza, 2020b).

- Operar correctamente los quemadores, manteniendo los parámetros establecidos para la explotación en cuanto a: flujo y presión de atomización; y temperatura de calentamiento, humedad y grado de preparación del combustible. 
- Disminuir el exceso de aire tanto como se pueda y mantenerlo en el valor óptimo durante la operación a cargas parciales.

- Maximizar el aprovechamiento del calor sensible disponible en los gases de la combustión, para lograr que su temperatura a la salida de las chimeneas sea lo más baja posible, sin que esto implique la condensación de los vapores de agua que contienen y la corrosión en los sistemas de circulación de agua y evacuación de los gases, lo cual provocaría incrementos en los costos de mantenimiento, roturas prematuras y baja disponibilidad de las instalaciones.

- Mantener limpias las superficies de transferencias de calor (por el interior y el exterior) y en buen estado técnico el aislamiento de las calderas, lo cual impactará positivamente en las dos mayores pérdidas existentes en el SGV $\left(q_{2}\right.$ y $\left.q_{5}\right)$.

- Hermetizar correctamente las paredes, registros y mirillas. No abrir estas últimas innecesariamente, para minimizar las infiltraciones de aire en las calderas de tiro balanceado (GV 2; 4 y 5) y las fugas de gases en la de horno presurizado (GV 6).

- Mantener la temperatura de la combustión en el valor adecuado, sin que disminuya o varíe significativamente, pues actúa como un catalizador positivo de la reacción y contribuye a maximizar la utilización de la exergía del calor de combustión ( $\left.E x_{\mathrm{Qc}}\right)$.

- Disminuir la diferencia de temperatura entre la sustancia de trabajo (el agua) y el agente de transformación (los gases producto de la combustión), para reducir al máximo el grado de irreversibilidad en los procesos de transferencia de calor y de transformación del agua en vapor. Aplicar varias etapas de calentamiento al agua de alimentación en los economizadores.

- Proyectar, mediante el análisis por técnicas numéricas, la simulación computacionaly otras herramientas de probada eficacia (Andrade, Quitiaquez y Toapanta, 2020); la implementación de tecnologías basadas en el uso de fuentes renovables de energía para el calentamiento del agua de alimentación suministrada a las calderas (Quitiaquez et al., 2020), lo cual reduciría la cantidad de combustibles fósiles quemados y mitigaría los impactos negativos ocasionados al medio ambiente.

Las acciones antes mencionadas constituyen el tercer aporte del presente trabajo. Su implementación integral en la CTE y el SGV, reduce las pérdidas de calor y eleva los rendimientos térmicos y exergéticos de las calderas. Los impactos positivos que generan en estas instalaciones no han sido suficientemente estudiados. Estos, conjugados con la optimización energética y operacional de las calderas, serán objeto de análisis a futuro.

\section{Conclusiones}

Se elaboró un algoritmo que sistematiza la metodología establecida para el balance termoexergético de calderas de vapor. El mismo sintetiza los procedimientos establecidos en trabajos precedentes, establece la secuencia de pasos a seguir y define los parámetros que se requieren para la evaluación térmica, por los métodos directo e indirecto, y exergética de los generadores de vapor estudiados y otros de similares características.

Se realizó un análisis termoenergético integral del sistema de generación de vapor de una termoeléctrica de $49 \mathrm{MW}$. La instalación tiene un elevado grado el aprovechamiento de la energía térmica. Sin embargo, es baja su capacidad de utilización de la exergía disponible, debido al deterioro de algunos de sus parámetros de operación y a las irreversibilidades inherentes a los 
procesos de combustión, transferencia de calor y de transformación del agua en vapor. Sus rendimientos, térmico y exergético, ascendieron a 90.106 y $45.491 \%$, respectivamente, y el grado de pérdida de exergía fue del $54.509 \%$.

Se evidenció que en el SGV se han deteriorado, respecto a los valores de diseño, los siguientes parámetros operacionales: producción, temperatura y presión del vapor sobrecalentado; temperatura del agua de alimentación y de los gases producto de la combustión; y la calidad del combustible. Las causas que originan esta afectación pueden ser mitigadas o eliminadas mediante la implementación de acciones científicas y técnico-organizativas, que contribuyan a incrementar los rendimientos térmicos y exergéticos de las calderas. Tales acciones impactan positivamente en indicadores energéticos, económicos y ambientales asociados al trabajo de las instalaciones. Este aspecto, integrado a la optimización energético-operacional de las calderas, será investigado con posterioridad.

\section{Bibliografía}

Nugroho Agung P., Ria L., Danar Susilo W., Valiant Lukad P., Maedanu F., Muhammad I., Lip Huat S., Renanto H. (2017). Exergy Analysis of Boiler Process Powered by Biogas Fuel in Ethanol Production Plant: a Preliminary Analysis. Energy Procedia, 142: 216-223. https://doi.org/10.1016/j.egypro.2017.12.035.

Andrade Cando, A. X.; Quitiaquez Sarzosa, W. y Toapanta, L. F. (2020). CFD Analysis of a Solar Flat Plate Collector with Different Cross Sections. Enfoque UTE, 11(2), pp. 95-108. doi: 10.29019/enfoque. v11n2.601

Annaratone, D. (2019). Steam Generator. Berlin: Springer.

Arshad, A.; Muhammad, H.; Habib, A. et al. (2019). Energy and Exergy Analysis of Fuel Cells: A Review. Thermal Science and Engineering Progress 9(-), p: 308-321. doi: 10.1016/j.tsep.2018.12.008

Bluestein, M. (2020). Thermodynamics and Heat Power. Boca Raton: Taylor \& Francis.

Borroto, A., y Rubio, Á. (2010). Combustión y generación de vapor. La Habana, Cuba: Editorial Félix Varela.

Buecker, B. (2012). Basics of Boiler and Heat Recovery Steam Generator Design. Oklahoma: Penn Well Corporation.

Camaraza, Y. (2017). Introducción a la termotransferencia. La Habana, Cuba: Editorial Universitaria.

Camaraza, Y. (2018). Introducción a la termotransferencia (2. ${ }^{\circ}$ ed.). La Habana, Cuba: Editorial Universitaria.

Camaraza, Y. (2020a). Transferencia de calor por conducción. La Habana, Cuba: Editorial Universitaria.

Camaraza, Y. (2020b). Transferencia de calor por convección (2. ed.). La Habana, Cuba Editorial Universitaria. La Habana, Cuba.

Çengel, Y. y Boles, M. (2014). Thermodynamics: An Engineering Approach, (8. ed.). McGraw-Hill.

Centeno-González, F. O.; Silva, E. E.; Villa, H. F. et al. (2017). CFD Modeling of Combustion of Sugarcane Bagasse in an Industrial Boiler. Fuel, 193, p. 31-38. doi: 10.1016/j.fuel.2016.11.105.

Cortés, M.; Cornes, Y.; Alomá. I. y González, E. (2019). Evaluación del sistema energético en el central azucarero Quintín Bandera. Centro Azúcar, 46(3), 66-78.

Edmonds, W. y Kennedy, T. (2017). An Applied Guide to Reseacrh Designs: Quantitative, Qualitative, and Mixed Methods (2. ${ }^{\circ}$ ed.). Los Ángeles, USA: SAGE Publications.

Flynn, D. (2013). Thermal Power Plant Simulation and Control. London: Springer.

Ganapathy, V. (2013). Industrial Boilers and Heat Recovery Steam Generators: Design, Applications and Calculations. New York: Marcel Dekker, Inc.

Harish, S., y Baldi, S. (2018). Monitoring Energy Efficiency of Condensing Boilers via Hybrid First Principle Modelling and Estimation. Energy, 142, 121-129. 
Hasnain, S.; Khurram, M.; Akhter, J.; Ahmed, B. y Abbas, N. (2020). Selection of an Industrial Boiler for a Soda-Ash Production Plant Using Analytical Hierarchy Process and Topsis Approaches. Case Studies in Thermal Engineering, 19(-) 100636, p:1-14 doi: 10.1016/j.csite.2020.100636

ISO International Organization for Standardization. (2018). ISO 50001 Energy Management Systems-Requirements with Guidance for Use. Geneva, Switzerland.

Javadi, M.; Hoseinzadeh, S.; Ghasemiasl, R.; Heyns, P. y Chamkha, A. (2019). Sensitivity Analysis of Combined Cycle Parameters on Exergy, Economic, and Environmental of a Power Plant. Journal of Thermal Analysis and Calorimetry, 13(3), p:1-10. doi: 10.1007/s10973-019-08399-y

Jiménez, J.; Jarquin, G.; Durán, M. y García, J. (2011). Análisis exergético del generador de vapor de 350 MW a cargas parciales. MACl, 3(-), 715-718.

Jiménez, R.; Madrigal, J.; Lapido, M. y Vidal, D. (2016). Método para la evaluación de la eficiencia e impacto ambiental de un GV. Ingeniería Energética, 37(2), 135-144.

Kitto, J. B. y Stultz, S. C. (2015). Steam, its Generation and Use. Ohio: The Babcock \& Wilcox Company.

Krasniqi-Alidema, D.; Filkoski, R. y Krasniqi, M. (2018). Exergy Efficiency Analysis of Lignite-Fired Steam Generator. Thermal Science 22(5), p. 2087-2101. doi: 10.2298/TSCI180131265

Labañino, N. (2015). Diagnóstico energético de calderas de vapor: estudio de casos. Tesis de Ingeniería, Facultad de Metalurgia y Electromecánica, Universidad de Moa, Cuba.

Martha de Souza, G. F. (2012). Thermal Power Plant Performance Analysis. London: Springer.

Pankratov, G. (1987). Problemas de Termotecnia. Moscú, URSS: Editorial MIR.

Patro, B. (2015). Efficiency Studies of Combinati on Tube Boilers. Alexandria Engineering Journal. p. 1-10. doi: 10.1016/j.aej.2015.12.007

Peduzzi, E.; Boissonnet, G. y Maréchal, F. (2016). Biomass modelling: Estimating Thermodynamic Properties from the Elemental Composition. Fuel, 181, p. 207-217. doi: 10.1016/j.fuel.2016.04.111.

Pérez, C.; Cordovés, A. y Terán, J. (2016). Design and Implementation of a Control System to Improve the Quality of the Combustion Gases in the Fire-tube Boiler of 5 BHP. Enfoque UTE, 7(2), 55-68. doi: 10.29019/enfoqueute.v7n2.95

Pérez-Sánchez, A.; Fernández, F. y González, R. (2017). Evaluación de la generación de vapor de la fábrica de refrescos 23 de agosto, en Camagüey. Mutis, 7(1), 7-19, doi: 10.21789/22561498.1198.

Quitiaquez, W.; Simbaña, l.; Isaza-Roldán, C. A. et al. (2020). Review of the State of Art of DX-SAHP Systems to Obtain Domestic Hot Water. Enfoque UTE, 11(2), 29-46. doi. 10.29019/enfoque.v11n2.565.

Rastogi, P.; Sharma, N. y Gupta, B. (2018). Energy and Exergy Analysis of Boiler in Bagasse Based 20 MW Steam Power Plants. Int. J. of Eng. and Tech., 4(2), 444-449.

Rodríguez-Ramos, A. y Llanes-Santiago, O. (2014). Diagnóstico de fallos en un GV BKZ-340-140-29M utilizando herramientas de lógica difusa. Ing. Mecánica, 17(2), 147-156.

Rubio, A. (2015). Generadores de vapor. Funcionamiento y explotación. Santa Clara, Cuba: Editorial Feijóo.

Tozlu, A.; Büyükmurat, Y. y Özahi, E. (2020). Thermoeconomic Analyses of an Actual Power Plant. Turkish Journal of Electromechanics \& Energy, 5(1), 9-15.

Valencia, G.; Piero, J. y Campos, J. (2019). Energy Optimization of Industrial Steam Boiler using Energy Performance Indicator. Int. J. of Energy Eco. and Policy, 9(6), 109-117.

Valles, A.; Acosta, L. y Pérez, A. (2014). Evaluación energética de los GV F1-2 y BH-109 de una refinería cubana de petróleo. Revista Especializada en Ingeniería, 8(-), 1-10.

Velázquez, A.; Corrales, J. y Pérez, L. (2019). Evaluación termoexergética del GV del campus Lenin de la Universidad de Las Tunas. Opuntia Brava, 12(1), 454-468. 